\title{
Pre-print of the paper:
}

Yuan, X. L., Zhang, D., Zhang, J.F., Zhang, M.Y., and Guedes Soares, C. (2020), "A novel collision risk awareness framework for ships in real-time operating conditions", Developments in the Collision and Grounding of Ships and Offshore Structures, C. Guedes Soares (Ed.), Taylor \& Francis Group, London, UK, pp. 337-343.

\section{A novel collision risk awareness framework for ships in real-time operating conditions}

\author{
X.L. Yuan \\ Intelligent Transportation Systems Research Center, Wuhan University of Technology, Wuhan, China; and, \\ College of Logistics Engineering, Wuhan University of Technology, Wuhan, China. \\ D. Zhang \& J.F. Zhang \\ National Engineering Research Center for Water Transport Safety, Wuhan University of Technology, Wuhan, \\ China; and, Intelligent Transportation Systems Research Center, Wuhan University of Technology, Wuhan, \\ China.
}

M.Y. Zhang

Department of Mechanical Engineering, School of Engineering, Aalto University, Finland.

C. Guedes Soares

Centre for Marine Technology and Ocean Engineering (CENTEC), Instituto Superior Técnico, Universidade de Lisboa, Lisbon, Portugal.

\begin{abstract}
Ship collisions sometimes occur at sea and a significant cause of ship collision accident is the lack of collision risk awareness. In order to quantify the collision risk of the ship in real operating conditions, a novel collision risk awareness framework is proposed for ships in real-time operating conditions. It mainly consists of four models/stages. Firstly, the ship motions are identified using turning points based on (Automatic Identification System) AIS data in real operating conditions. Secondly, the uncertainty of ship motion patterns is analysed that the target ship is regarded as velocity obstacles. Then, the collision risk probabilistic model is utilized to assess the collision risk level in different areas based on the non-linear velocity obstacles algorithm. Finally, some case studies are carried out based on the proposed ship collision risk awareness framework. In the collision risk awareness framework, the dynamic and uncertainty characteristics of ship motion patterns in real operating conditions are considered, which could benefit on reducing the ship collision accident and improving the development of theoretical technology on intelligent collision avoidance decision making.
\end{abstract}




\section{INTRODUCTION}

Shipping has been a significant mode of transportation for domestic and international trade due to its large capacity and environment-friendly nature. Meanwhile, the consequence and occurrence frequency of ship collisions are not desirable. As a result, research on ship collision and anti-collision strategy has been an essential topic of maritime transportation study (Wang, et al. 2013). However, most research has many assumptions and are applied to specific scenarios, such as relatively simple dynamics of the ship. These bring some limitations in real applications to the crew assistance to make an anti-collision decision when facing with collision situations (Huang, et al. 2019).

Most of the systems cannot provide with a general picture in a complex situation showing all possibilities of manoeuvre in relation to changes of course and/or speed by the threatened vessel and taking account of all other vessels in the vicinity. By doing so, each obstacle in the vicinity of the vessel is represented by one shaded zone, and navigators can easily assess in respect to each fixed or moving obstruction in his vicinity based on the 'room-to-manoeuvre' concept.

This is the origin of Velocity Obstacle (VO) algorithms, and it can reflect a series of complex encounter situations, i.e., Do I have collision risk? If so, should I take avoiding action? If so, when and what are the possible alterations of course or speed, with regard to all vessels in the vicinity? (Degré, \& Lefèvre 1981). The VO algorithm can present a clear understanding of the collision risk during navigation. Moreover, it has been widely used in Unmanned Surface Vehicles (USVs) (Liu, et al. 2016), and multi-agent navigation.

Risk awareness makes a significant impact on anti-collision and is one of the most effective ways to identify and reduce the risks of maritime transportation in a sea area and to find the most effective ways to manage the risk (Perera, et al. 2010, Zhang, et al. 2016a). The main objective of this paper is to carry out a novel risk awareness framework based on VO algorithms for ship collision.

The rest of the paper is organized as follows: Section 2 provides a literature review of research on collision risk awareness with the focus on techniques and algorithms. In Section 3, a novel collision risk awareness framework is proposed, which contains ship action identification, the uncertainty of ship motion and collision risk probabilistic model based on the non-linear velocity obstacles algorithm. Section 4 presents a case study to verify the framework and methods. Discussion and conclusions on the results of the research and some limitations are discussed in Section 5 and Section 6.

\section{LITERATURE REVIEW}

\subsection{Anti-collision strategies and techniques}

Generally, ship anti-collision research can be categorized into three classes: prior anti-collision, process anti-collision, and post-anti-collision. Prior anti-collision studies are strategies and techniques made in advance. Prediction of accident consequences is proposed for Tianjin port using a Bayesian belief network model (Zhang, et al. 2016). Statistical analyses of maritime accidents data (Bye, \& Aalberg 2018) and AIS data are used to predict collision risk. A prediction model is provided of the ship collision risk based on classification and regression trees (Li, et al. 2018). Multiple linear regression model (Yim, et al. 2018) and empirical and semi-analytical methods (Liu, et al. 2018) are carried out for ship anti-collision. Another anti-collision strategy is to get information from the known safe route and use them to predict a possible route in the same area. For instance, some studies proposed an improved Douglas-Peucker (DP) algorithm considering the shape of vessel trajectory derived from course information of track points to generalize new route for ships.

Process anti-collision is a time-varying strategy via changing course or reduce speed along with all the route (Perera \& Guedes Soares 2015, Wang, et al. 2013). Also, some research establishes a whole system for navigating guidance. (Perera, et al. 2015) proposed an intelligent guidance system consisting of a navigation and control platform and a vessel model. (Park, et al. 2017) presented a probability flow method considering time-varying ship trajectory uncertainties for evaluating the collision risk between two moving surface ships.

Post anti-collision techniques make a conclusion about the characteristics of collision area, i.e. a series of hot spots that experienced high frequencies of vessel conflicts in the Sabine-Neches Waterways were identified through investigating the frequency of vessel conflict (Wu, et al. 2016, Wu, et al. 2018), also in the Strait of Istanbul (Altan, et al. 2018). In Singapore Strait, three ship collision risk indices were proposed to quantitatively assess the most risky legs in the Strait: index of speed dispersion, degree of acceleration and deceleration, and the number of fuzzy ship domain overlaps $(\mathrm{Qu}$, et al. 2011).

Other studies calculate the distance between two vessels on collision courses to evaluate the conditions among ships (Montewka, et al. 2010). The distance at the closest point of approach (DCPA) and the time to the closest point of approach (TCPA) 
are the two most important parameters to evaluate collision risk. However, they are not sufficient for estimating ship collision risk and for planning evasive manoeuvres. Their limitation was the lack of analytic solutions that would make it possible to efficiently use ship domains in real-time systems when the issue of computational time is taken into consideration. Some analytic formulas have been proposed for domain-based collision risk parameters, such as the degree of domain violation (DDV) and time to domain violation (TDV) (Szlapczynski, \& Szlapczynska 2016).

\subsection{Collision risk analysis method and VO algorithm}

In many related literature, previous research components of ship collision risk analysis have been carried out (Ulusçu, et al. 2009, Zhang, et al. 2015a). Extensive researches have been carrying out in the field of ship collision risk awareness (Shu, et al. 2018), some works have been done to prevent serious damage such as optimal control, (Kozynchenko, et al. 2018), nonlinear model predictive control (Abdelaal, et al. 2018) and information fusion (Borkowski 2017).

Ship safety domain is a term which is widely used in research on collision avoidance (Szlapczynski, et al. 2018). A circular ship domain is often adopted to quantify collision risk and simplify computation. To measure the collision risk from the ship action perspective, the VO technique is utilized. The idea of $\mathrm{VO}$ is to project the spatiotemporal relationship between a ship pair involved into encounter situation (relative distance, speed and course) into one ship's velocity domain, based on which the collision risk can be calculated by checking whether the speed of the ship falls into this velocity obstacle zone (Chen, et al. 2018). The ship's velocity falling into this velocity obstacle zone indicates that the two ships might result in a collision.

Considering that it is not practical to assume the ship to be sailing in a straight line with constant speed, the NL-VO algorithm is utilized as a basis to analyse the collision risk at different encounter stages. VO algorithms have been employed to various vehicles for collision prevention. Many types of VO algorithms are adopted to prevent collision with target ships whose motions might change over time: Linear-VO, Non-Linear VO, and probabilistic VO algorithms (Huang, et al. 2019). Both the obstacle avoidance and the goal approaches are considered, (Song, et al. 2018) introduced a two-level dynamic obstacle avoidance algorithm by combining the VO algorithm with the improved artificial potential field (APF) method in a non-emergency situation. Also, a Time Discrete
Non-linear Velocity Obstacle (TD-NLVO) method for collision candidate detection, which is based on the Non-linear Velocity Obstacle algorithm to detect collision candidate (Chen, et al. 2018). Moreover, VO can be used to determine ship domain, near-miss ships and trajectory planning.

\section{METHODOLOGIES}

\subsection{Risk awareness framework for ship collision}

In order to show real time collision risk for manoeuvres, a risk awareness framework with respect to ship action identification and ship action uncertainty is constructed. Firstly, the ship motions are identified using turning points based on AIS data in real operating conditions. Secondly, the uncertainty of ship motion patterns is analysed that the target ships are regarded as velocity obstacles. Then, the collision risk probabilistic model is utilized to assess the collision risk level in the different area based on the non-linear velocity obstacles algorithm. Fig. 1 presents the framework of collision risk awareness using non-linear velocity obstacle (NL-VO) algorithm approach.

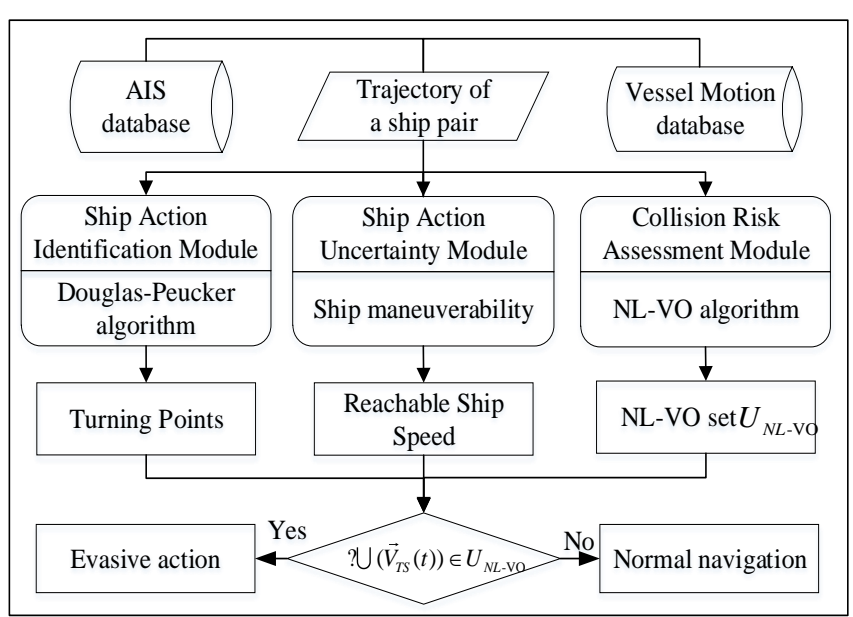

Figure 1. The framework of collision risk awareness

\subsection{Ship action identification model}

Ship action is a complex system as multiple factors have impact on it. The ship action identification problem can be simplified as determining the range of position, as shown in Figure 2. 


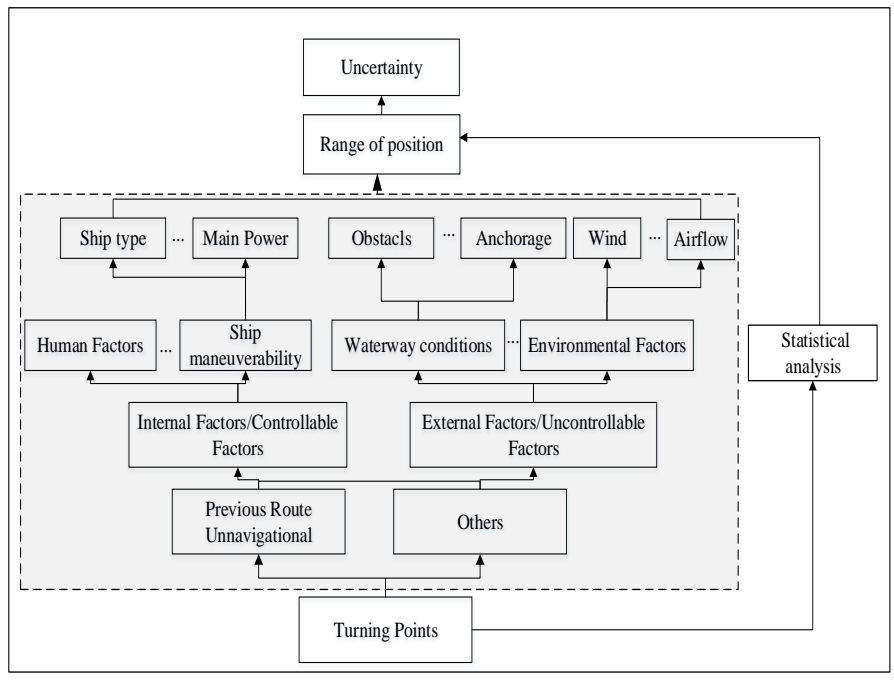

Figure 2. Schematic sketch of ship action identification model

The DP algorithm is one of the appropriate approaches in dealing with this problem as it can show different patterns based on threshold alteration. The algorithm can control the compression quality by means of the threshold, which is commonly used in simplifying the trajectory of moving objects (Zhang, et al. 2015b, Zhao, et al. 2019). In this paper, the threshold is used to display the range of position as shown in Figure 3.

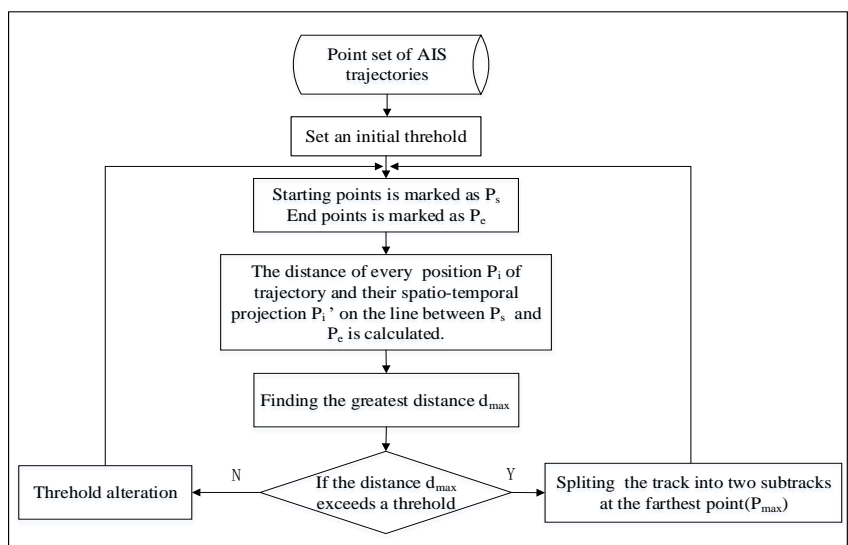

Fig 3. Improved DP algorithm theory

The AIS trajectory can be marked as a point set $D=$ $\left\{P_{1}, \ldots, P_{i}\right\}$ according to the DP theory. Threshold alteration is a critical element showing the range of course and it changes over factors that include ship type, navigating waterway and environmental conditions. In order to calculate the distance of point-to-line, geographical coordinates should be converted into the coordinates in Mercator projection (Zhang, et al. 2015b). The coordinates in Mercator projection of the track point $(X, Y)$ are calculated as follows:

$$
\begin{aligned}
& r_{o}=\frac{a \cos \varphi_{o}}{\sqrt{1-e_{o}^{2}} \sin ^{2} \varphi_{o}} \\
& q=\ln \left(\tan \left(\frac{\pi}{4}+\frac{\varphi}{2}\right)\left(\frac{1-e \sin \varphi}{1+e \sin \varphi}\right)^{e / 2}\right)
\end{aligned}
$$

$$
\begin{aligned}
& X=r_{0} \times \lambda \\
& Y=r_{0} \times q
\end{aligned}
$$

where $\varphi$ and $\lambda$ denote the latitude and longitude. $\varphi_{0}$ represents the standard latitude in Mercator projection; $a$ represents the long radius of earth ellipsoid. $e$ represents the first eccentricity of earth ellipsoid. $r_{0}$ represents the radius of parallel circle of standard latitude. $q$ represents the isometric latitude. The point-to-line distance can be calculated through vector method, which is shown as

$d=\frac{|a \times b|}{a}$

where $a$ denotes the vector from the start point of line segment to the end point of line segment; $b$ denotes the vector from the start point of line segment to the target point. The detail derivation process is elaborated in (Zhao, et al. 2018). Then a threshold interval can be calculated that can help forecast the risk probability.

\subsection{Ship action uncertainty model}

Prior anti-collision is an important part of anti-collision strategy network, and effective ship collision risk detection, prediction and awareness are required for operators to make appropriate decisions for evasive manoeuvres. The first step is to understand ship action uncertainty. Ship action uncertainty is affected by environmental and operational factors. In this paper, the focus is mainly on the velocity and course, aiming at trying to make a probabilistic evaluation of ship action. Ship action uncertainty model can be expressed as:

$$
I_{i}=\left\{V_{i}, C_{i}\right\}
$$

where $V_{i}, C_{i}$ refers to velocity and course forming action uncertainty model of ship $i . I_{i}$ is a ship action uncertainty set. The contribution of Non-linear velocity obstacle (NL-VO) algorithm is regarding ship action as a non-linear motion. The speed of navigating ships changes over time because of human action, structure of ships, regulations and environment. As a critical element in NLVO model, speed distribution plays an important role on ship action uncertainty recognition. In some waterways, it can be found that the same type of ships almost have the same pattern of speed. The speed of ships varies within a certain interval. This means that most of the ships do not navigate at full speed and the Gaussian Model can effectively express the situation. As a result, the speed distribution can be expressed as: 
$f(\mathrm{v})=\frac{1}{\sqrt{2 \pi \sigma_{v}^{2}}} \exp \left\{-\frac{\left(x-u_{v}\right)^{2}}{2 \sigma_{v}^{2}}\right\}$

where $u_{v}$ is the sample mean, $\sigma_{v}$ is the standard deviation. The $\mathrm{x}$-axis is the velocity, and the $\mathrm{y}$-axis is the frequency, and so as the distribution of course.

$$
f(\mathrm{c})=\frac{1}{\sqrt{2 \pi \sigma_{c}^{2}}} \exp \left\{-\frac{\left(x-u_{c}\right)^{2}}{2 \sigma_{c}^{2}}\right\}
$$

\subsection{Non-linear NL-VO algorithm approach}

The NL-VO set is the collection of all the conflicting velocities leading to the ship collision. The basic idea of NL-VO algorithm is to identify the NL-VO set that a collision risk occurs in the near future if the give-way ship's speed falls into this set.

$$
\left\{\begin{array}{l}
U_{N L_{-} V O(t)}=\bigcup_{\mathrm{t}}^{\infty}\left(\frac{P_{i}(t)-P_{j}\left(t_{0}\right)}{\left(t-t_{0}\right)} \oplus \frac{\operatorname{ConfP}(O, R)}{\left(t-t_{0}\right)}\right) \\
\operatorname{Conf} P(O, R)=\left\{\left\|P_{i}(t)-P_{j}\left(t_{0}\right)\right\| \leq R\right\}
\end{array}\right.
$$

where $U_{N L_{-} v o(t)}$ is the NL-VO set. $P_{i}$ and $P_{j}$ are the position of the target ship and own ship respectively. ConfP is the term describing all the possible positions of the target ship when ship collision risk occurs between own ship and target ship at time $t$. $\oplus$ is Minkowski addition, and the prohibited region around the own ship can be formulated as $P_{i} \oplus \operatorname{Conf} P(O, R)$. If the speed vector of the target ship $\vec{V}$ is the element of $U_{N L_{-} V o(t)}$, the ship collision risk exists. The formula derivation process is elaborated in (Huang, et al. 2019).

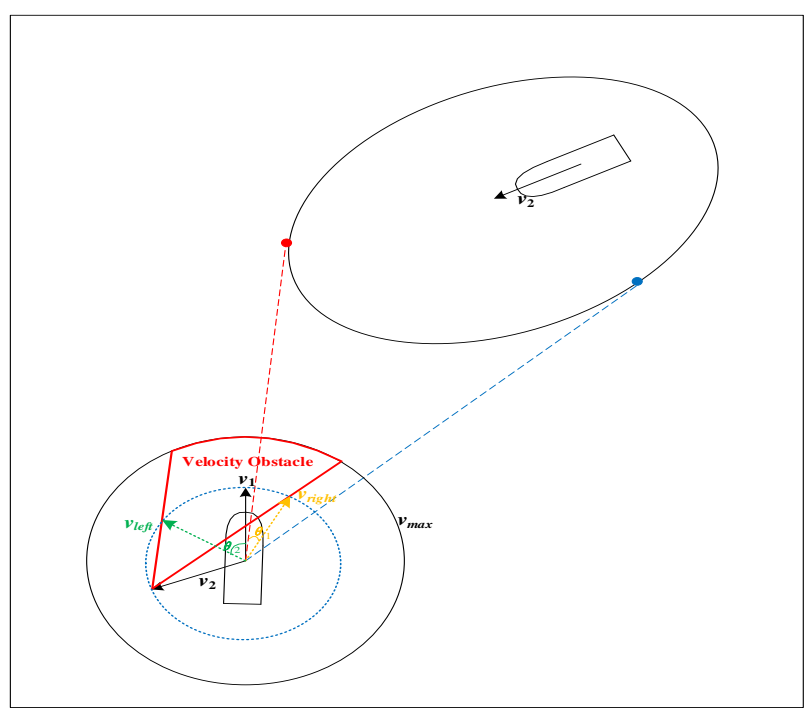

Figure 4. Schematic sketch of the NLVO algorithm

The $\partial$ denotes the range of position calculated from ship action identification model. Then $\partial_{i}, \partial_{j}$ are the range of the target ship and own ship respectively. The OS is regarded as a reference, then
$P_{j}\left(t_{0}\right)=\left[\begin{array}{l}0 \\ 0\end{array}\right]$. Then Equation (9) can be further

described as:

$$
U_{N L_{-} V O(t)}=\bigcup_{\mathrm{t}}^{\infty}\left(\frac{\partial_{i}}{\left(t-t_{0}\right)} \oplus \frac{\operatorname{Conf} P(O, R)}{\left(t-t_{0}\right)}\right)
$$

\section{CASE STUDY}

\subsection{Data Source}

Based on the risk awareness framework, an accident report is taken as a case study. An appropriate dynamic threshold can be obtained in order to show collision details and some anti-collision decisions can be made to achieve risk awareness. Two ships collided in the East China Sea and the information of ship speed, position and course was retrieved from AIS between 19:20'07' to 20:04'48', Jan.6th 2018. Outliers and some pre-processing have been done to get useful information. The longitude and latitude are transformed in meters in Mercator projection as shown in Figure 5.

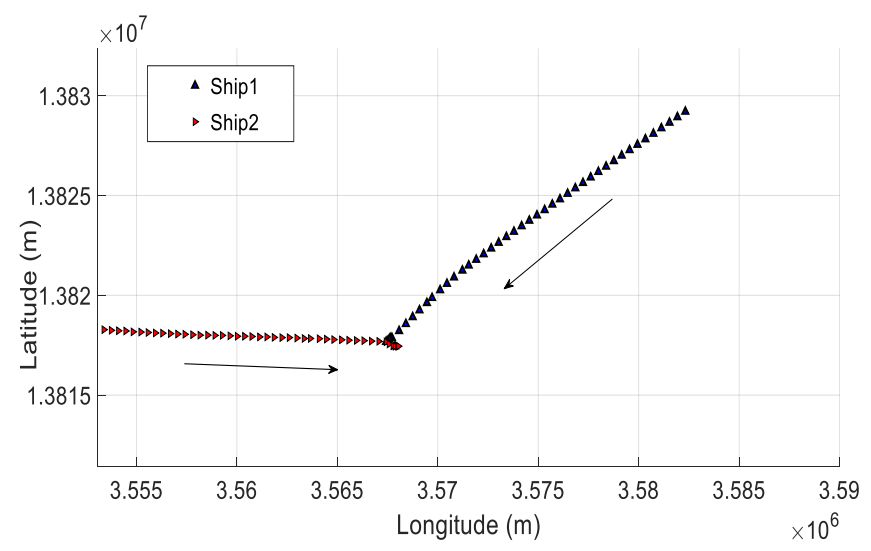

Figure 5. Trajectories of collision ships

\subsection{Ship action identification}

The range of position can be used to show the uncertainty of ship action with dynamic threshold. The threshold is influenced by many factors, such as characteristics of navigation area, speed, ship type and trajectories. In this paper, two accident ships containing 45 pieces of AIS data is studied. The threshold step is determined with $5 \mathrm{~m}$ according to AIS data. Meanwhile, ship domain of $2 \mathrm{n}$ miles and double ship domain is selected for early detecting collision risk. It can be found that a critical point that records decrease sharply. This threshold can be used to express the action of ships. Then performance results of compression by the DP algorithm of trajectory 1 is illustrated Figure 6. 


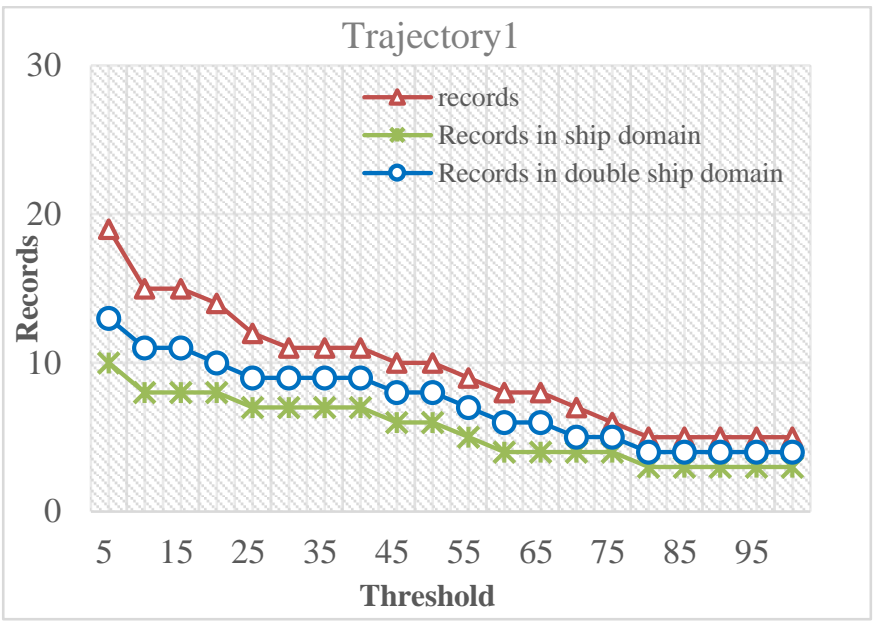

Figure 6. Relationships between dynamic threshold and records of trajectory 1

According to Figure 6, a critical point can be pointed out when the threshold is 10 . It indicates that this threshold can significantly show the action of trajectory 1 . The reason why both ship domains and double ship domains are analysed is that it can make a further understanding of the threshold's impact on the whole trajectory and collision part. Their tendency is almost the same. So the threshold of $10 \mathrm{~m}$ can be used to show the action of ship and the critical details of collision parts. The same idea can be used in trajectory 2 as shown in Figure 7.

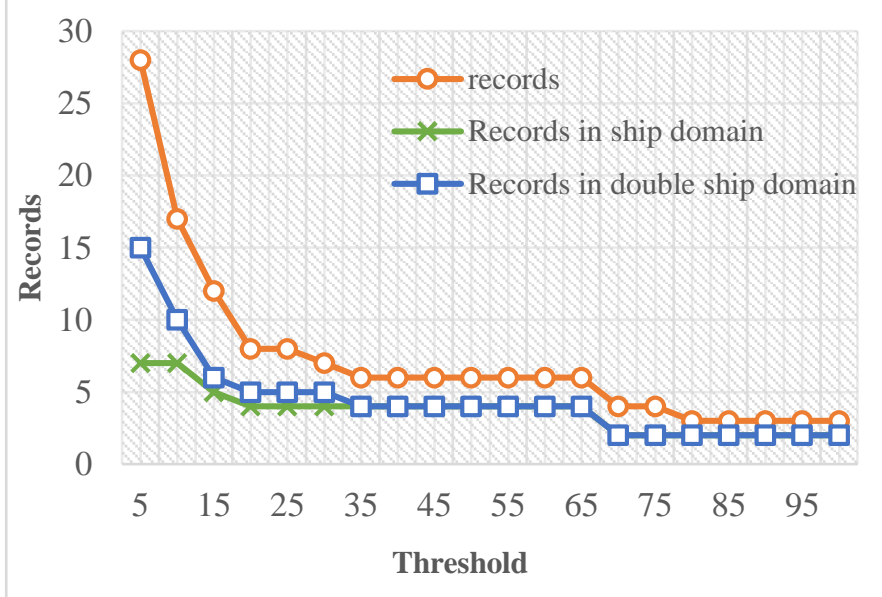

Figure 7. Relationships between dynamic threshold and records of trajectory 2

\subsection{Ship action uncertainty}

A Gaussian Model can effectively express the ship action uncertainty (Rong, et al. 2019). For trajectory 1 , the sample mean $u_{v 1}$ is $13.2 \mathrm{kn}$, the standard deviation $\sigma_{v 1}$ is 0.43 . For trajectory 2 , the sample mean $u_{v 2}$ is $8.2 \mathrm{kn}$, the standard deviation $\sigma_{v 2}$ is 0.41 . So the speed distribution can be determined and the histogram and Gaussian distribution are presented in Figure 8 and Figure 9.

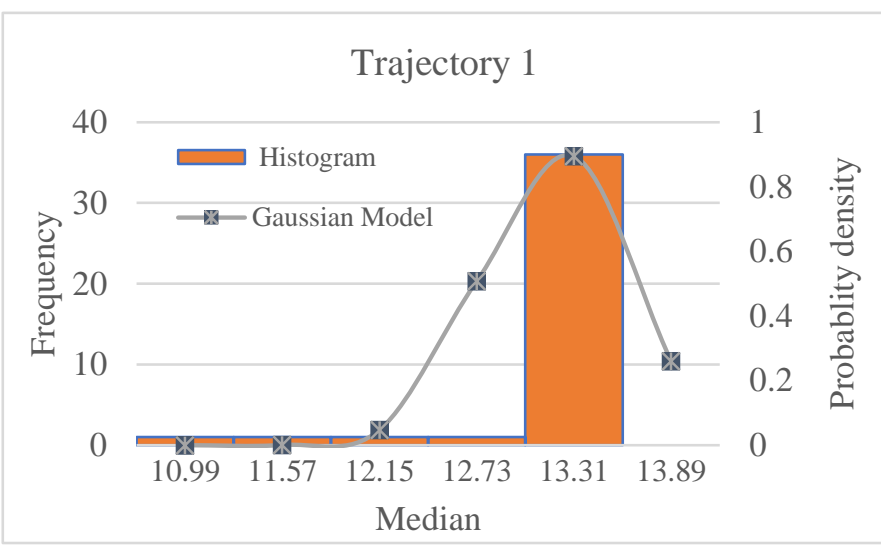

Figure 8. Distribution of trajectory 1

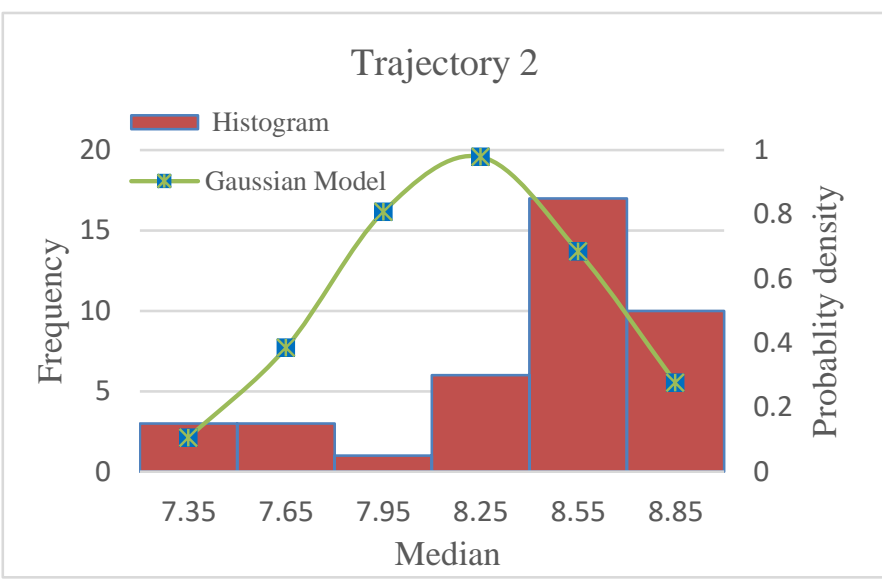

Figure 9. Distribution of trajectory 2

\subsection{NL-VO algorithm}

According to risk awareness model and AIS data of two ships, velocity obstacle is determined based on DCPA and TCPA. The red circular sector in Figure 10 represents velocity obstacle with two ships approaching each other.

In order to make the collision avoidance strategy, velocity and course distribution and their interval can be estimated considering ship types, navigational time and navigational area. The collision avoidance process is presented in Figure 11 and Figure 12.

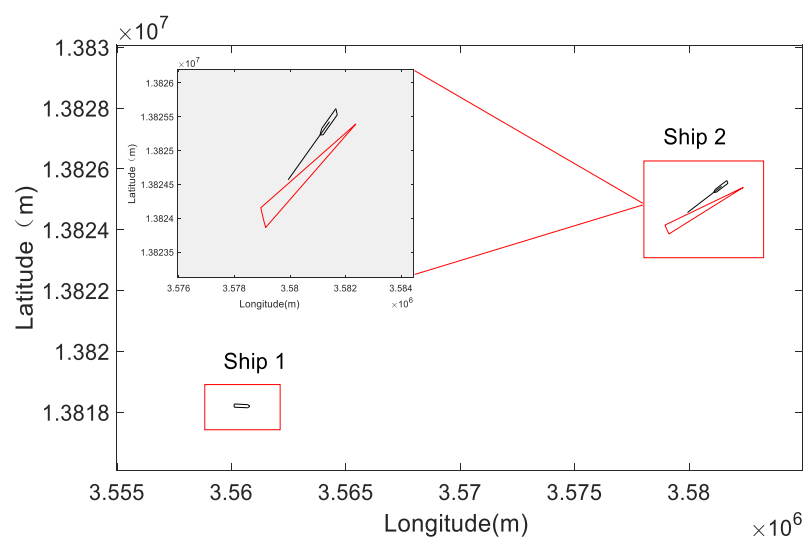

a (Time stamp: 19:21:07) 


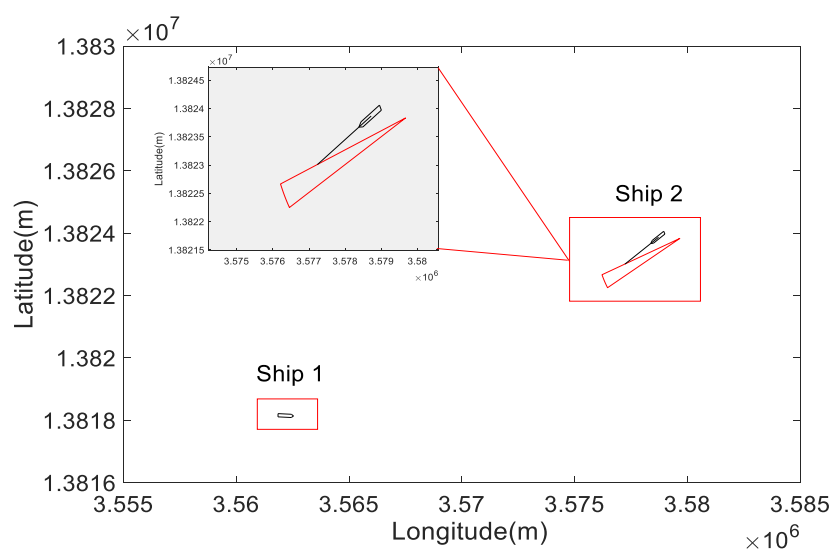

b (Time stamp: 19:27:07)

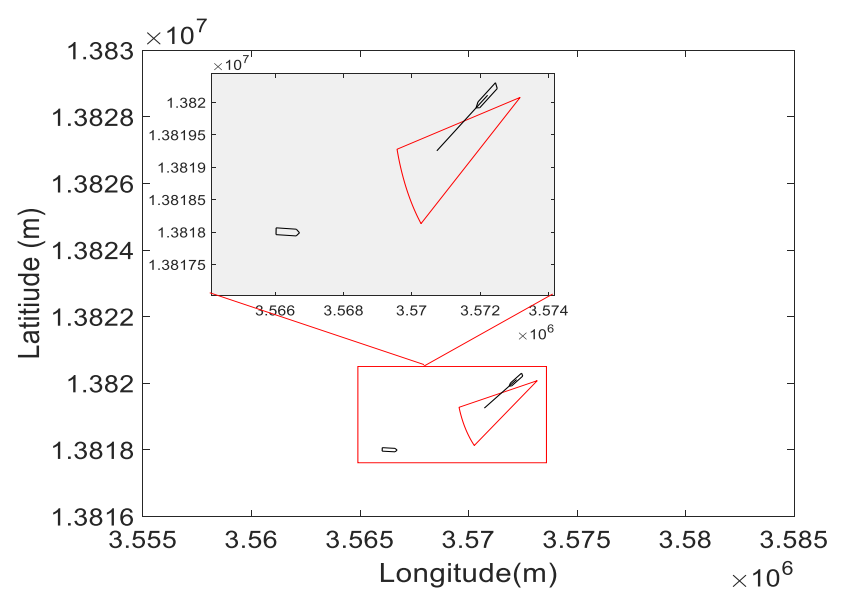

c (Time stamp: 19:46:07)

Figure 10 . Velocity obstacle

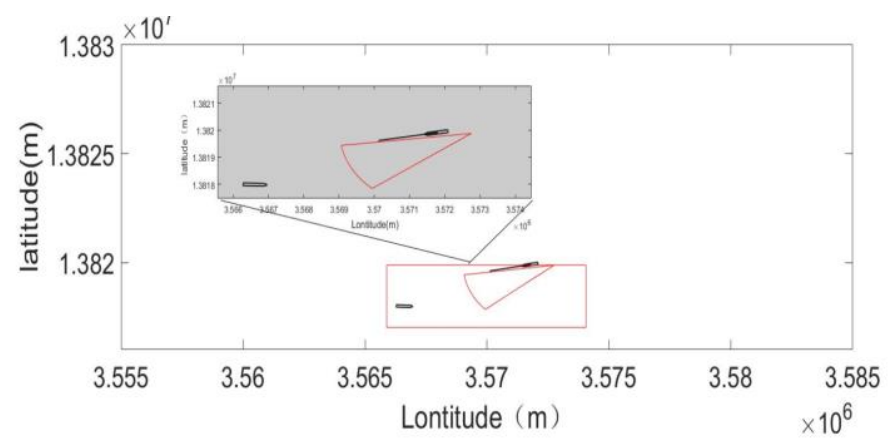

Figure 11. anti-collision strategy

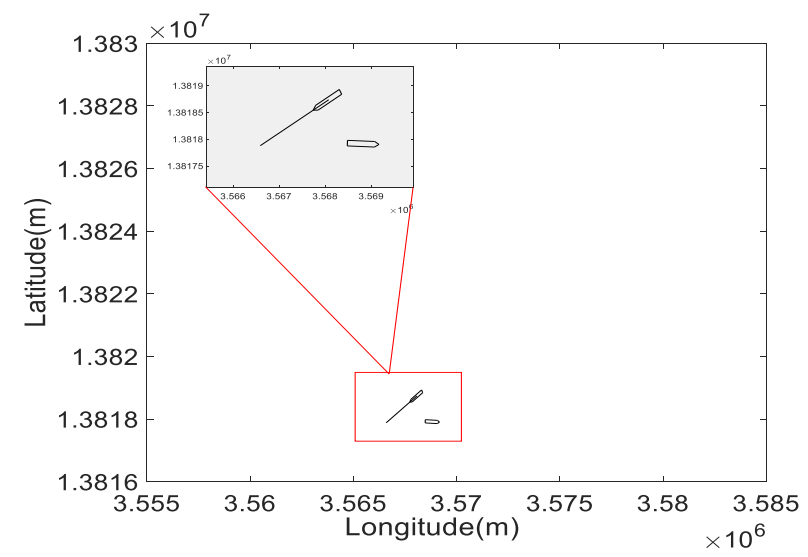

Figure 12. Collision avoidance

\section{DISCUSSION}

The NLVO method can help manoeuvres in real time conditions. One can obtain a safe area limited by manoeuvrability except a VO circular sector. Any vector in the safe area will not collide with other dynamic or static obstacles. The case study raised in this paper is from an accident report. Two ships collide with each other and cause heavy damage on environmental pollution and human loss. The method discussed in this paper can give deck officers a clear risk awareness about the risk changing with ships approaching each other. Eventually, when facing complicated encounter situations such as multi-motion vessels and sandbanks, deck officers can quickly make decisions about how to manoeuvre. This framework and method can also be applied in intelligent or unmanned ships. Machines can control the ship autonomously or independently. So the methods can be expanded in modern ships.

\section{CONCLUSIONS}

Risk awareness is one of the most effective ways to identify and reduce the risks of maritime transportation in sea area. However, risk awareness is complicated because of many influencing factors that are difficult to be quantified. The paper proposes a collision risk awareness framework in order to reduce the awareness uncertainty and errors by learning from historical data. The risk awareness framework consists of four models or stages. Firstly, the ship motions are identified using turning points by DP algorithm based on AIS data. Secondly, the uncertainty of ship motion patterns is analysed. Then, the collision risk probabilistic model is utilized to assess the collision risk level in the different area based on the non-linear velocity obstacles algorithm. Finally, an accident occurred in the East China Sea is used to verify this method. The results show that this framework can be used to make a risk awareness in real conditions. The framework proposed in this paper is innovative and provides a new perspective for collision avoidance. Multiple researches will be done according to this framework in the future research. However, there exist certain limitations that require further research efforts. For instance, the NLVO model is established based on DCPA and TCPA, and their limitations in collision avoidance have been studied by some researches. Also, the AIS transceivers positions in the case study are not considered, and the accuracy of result will be affected. So improvements on NLVO model and AIS transceivers positions will be further addressed. 


\section{ACKNOWLEDGEMENTS}

The research was supported by the National Key Research and Development Project (2017YFC0804900, 2017YFC0804904), National Key Technologies Research \& Development Program (2017YFE0118000), and the Marie Skłodowska Curie grant agreement No. 730888 (RESET).

\section{REFERENCES}

Abdelaal, M., Fränzle, M. \& Hahn, A. 2018. Nonlinear Model Predictive Control for trajectory tracking and collision avoidance of underactuated vessels with disturbances. Ocean Engineering 160: 168-180.

Altan, Y.C. \& Otay, E.N. 2018. Spatial mapping of encounter probability in congested waterways using AIS. Ocean Engineering 164: 263-271.

Borkowski, P. 2017. The Ship Movement Trajectory Prediction Algorithm Using Navigational Data Fusion. Sensors 17: 32-43.

Bye, R.J. \& Aalberg, A.L. 2018. Maritime navigation accidents and risk indicators: An exploratory statistical analysis using AIS data and accident reports. Reliability Engineering \& System Safety 176: 174-186.

Chen, P., Huang, Y., Mou, J. \& van Gelder, P.H.A.J.M. 2018. Ship collision candidate detection method: A velocity obstacle approach Ocean Engineering 170: 186-198.

Degré, T. \& Lefèvre, X. 1981. A Collision Avoidance System. Journal of Navigation 34: 294-302.

Huang, Y., Chen, L. \& van Gelder, P.H.A.J.M. 2019. Generalized velocity obstacle algorithm for preventing ship collisions at sea. Ocean Engineering 173: 142-156.

Kozynchenko, A.I. \& Kozynchenko, S.A. 2018. Applying the dynamic predictive guidance to ship collision avoidance: Crossing case study simulation. Ocean Engineering 164: 640-649.

Li, Y., Guo, Z., Yang, J., Fang, H. \& Hu, Y. 2018. Prediction of ship collision risk based on CART. IET Intelligent Transport Systems 12: 1345-1350.

Liu, B., Pedersen, P.T., Zhu, L. \& Zhang, S. 2018. Review of experiments and calculation procedures for ship collision and grounding damage. Marine Structures 59: 105-121.

Liu, Z., Zhang, Y., Yu, X. \& Yuan, C. 2016. Unmanned surface vehicles: An overview of developments and challenges. Annual Reviews in Control 41: 71-93.

Montewka, J., Hinz, T., Kujala, P. \& Matusiak, J. 2010. Probability modelling of vessel collisions. Reliability Engineering \& System Safety 95: 573-589.

Park, J. \& Kim, J. 2017. Predictive Evaluation of Ship Collision Risk Using the Concept of Probability Flow. IEEE Journal of Oceanic Engineering 42: 836-845.

Perera, L.P., Carvalho, J.P. \& Guedes Soares, C. 2010. Fuzzy logic based decision making system for collision avoidance of ocean navigation under critical collision conditions. Journal of Marine Science and Technology 16: 84-99.

Perera, L.P., Ferrari, V., Santos, F.P., Hinostroza, M.A. \& Guedes Soares, C. 2015. Experimental Evaluations on Ship Autonomous Navigation and Collision Avoidance by Intelligent Guidance. IEEE Journal of Oceanic Engineering 40: 374-387.

Perera, L.P. \& Guedes Soares, C. 2015. Collision risk detection and quantification in ship navigation with integrated bridge systems. Ocean Engineering 109: 344-354.
Qu, X., Meng, Q. \& Suyi, L. 2011. Ship collision risk assessment for the Singapore Strait. Accident Analysis \& Prevention 43: 2030-2036.

Rong, H., Teixeira, A.P. \& Guedes Soares, C. 2019. Ship trajectory uncertainty prediction based on a Gaussian Process model. Ocean Engineering 182: 499-511.

Shu, Y., Daamen, W., Ligteringen, H., Wang, M. \& Hoogendoorn, S. 2018. Calibration and validation for the vessel maneuvering prediction (VMP) model using AIS data of vessel encounters. Ocean Engineering 169: 529-538

Song, L., Su, Y., Dong, Z., Wei, S., Xiang, Z. \& Mao, P. 2018. A two-level dynamic obstacle avoidance algorithm for unmanned surface vehicles. Ocean Engineering 170.350-361:

Szlapczynski, R., Krata, P. \& Szlapczynska, J. 2018. Ship domain applied to determining distances for collision avoidance manoeuvres in give-way situations. Ocean Engineering 165: 43-54.

Szlapczynski, R. \& Szlapczynska, J. 2016. An analysis of domain-based ship collision risk parameters. Ocean Engineering 126: 47-56.

Ulusçu, Ö.S., Özbaş, B., Altıok, T. \& Or, İ. 2009. Risk Analysis of the Vessel Traffic in the Strait of Istanbul. Risk Analysis 29: 1454-1472.

Wang, Y., Zhang, J., Chen, X., Chu, X. \& Yan, X. 2013. A spatial-temporal forensic analysis for inland-water ship collisions using AIS data. Safety Science 57: 187-202.

Wu, X., Mehta, A.L., Zaloom, V.A. \& Craig, B.N. 2016. Analysis of waterway transportation in Southeast Texas waterway based on AIS data. Ocean Engineering 121: 196-209.

Wu, X., Rahman, A. \& Zaloom, V.A. 2018. Study of travel behavior of vessels in narrow waterways using AIS data A case study in Sabine-Neches Waterways. Ocean Engineering 147: 399-413.

Yim, J., Kim, D. \& Park, D. 2018. Modeling perceived collision risk in vessel encounter situations. Ocean Engineering 166: 64-75.

Zhang, J., Teixeira, Â.P., Guedes Soares, C., Yan, X. \& Liu, K. 2016. Maritime Transportation Risk Assessment of Tianjin Port with Bayesian Belief Networks. Risk Analysis 36: 1171-1187.

Zhang, J., Zhang, D., Yan, X., Haugen, S. \& Guedes Soares, C. 2015a. A distributed anti-collision decision support formulation in multi-ship encounter situations under COLREGs. Ocean Engineering 105: 336-348.

Zhang, S., Liu, Z., Cai, Y., Wu, Z. \& Shi, G. 2015b. AIS Trajectories Simplification and Threshold Determination. Journal of Navigation 69: 729-744.

Zhao, L. \& Shi, G. 2018. A method for simplifying ship trajectory based on improved Douglas-Peucker algorithm. Ocean Engineering 166: 37-46.

Zhao, L. \& Shi, G. 2019. A trajectory clustering method based on Douglas-Peucker compression and density for marine traffic pattern recognition. Ocean Engineering 172: 456-467. 